(c) 2011 IEEE. Personal use of this material is permitted. Permission from IEEE must be obtained for all other users, including reprinting/ republishing this material for advertising or promotional purposes, creating new collective works for resale or redistribution to servers or lists, or reuse of any copyrighted components of this work in other works.

\title{
SIMULATION MODELING OF TOOL DELIVERY SYSTEM IN A MACHINING LINE
}

\author{
Benny Tjahjono \\ John Ladbrook \\ Cranfield University \\ Manufacturing Department \\ Building 50 School of Applied Sciences \\ Cranfield, Bedford MK43 0AL, UK
}

\begin{abstract}
This paper describes an industrial project aiming to enhance the existing simulation modeling suites used at a car engine factory in the UK. The company continues to enhance its simulation modeling capabilities towards so called the 'total plant modeling' which not only covers the production facilities but also key ancillary facilities. Tool delivery is one such ancillary process. The existing modeling practices at the company are limited to modeling tool changes and assume that tools meet their expected life and the replacement is always available. In reality, the tools are not always reaching the expected life, the facilities in the tool crib are a limiting resource and the tool inventory has to be minimized. The tool delivery system developed in this project has specific features that model how the tool crib operates, how tools are supplied to the machining lines and various operating strategies.
\end{abstract}

\section{INTRODUCTION}

Simulation modeling has been recognized as a key technological capability to support continuous improvement for manufacturing operations (Baines and Kay 2002). The company where this study is based has used simulation modeling as a decision making aid particularly in designing its production facilities worldwide. The existing factory simulation suites used at the company are typically easy to use and user friendly allowing the manufacturing engineers to build the detailed models of their production lines with minimal or almost no training needed. However, recently there is an increased demand on the speed and accuracy of the simulation results upon which the operational and managerial judgment will be based. Due to this pertinent demand, the simulation suites are continually being improved. The company would like to enhance the simulation modeling suites to cover so called the 'total plant modeling', which not only includes the main production facilities, such as machining and assembly lines, but also ancillary facilities that impact production.

Tool delivery is one such ancillary process within machining lines. Although the existing machining line simulation models are sufficiently detailed and complex, they have limitations in modeling the tool usage. In this respect, tools (e.g., drills, lathes, etc) are assumed to meet their expected life with the tool replacement (as well as the resources required to replace the tools) always being available when needed. In reality, the process is far more complex. The 'total plant modeling' intends to better model the whole production facility by incorporating the tool delivery process that includes strategies to minimize stoppages during the tool change, operations inside the tool crib, and delivery of the tools to the machining lines.

Inside the tool crib, new tools are stocked and used tools are serviced to be reused in the future. Servicing worn tools involves dismantling them from the tool-holders, cleaning and regrinding. Once returned to the crib, the reused cutting tools are then re-set, gauged and stored in the tool crib warehouse. 


\section{Tjahjono and Ladbrook}

The demand for the replacement tools is triggered from CNC machines by means of signaling, akin to the kanban cards in the 'just-in-time' system (Ohno 1988). The receipt of the signal from a CNC machine indicates that a new tool is needed, notifying the tool crib to prepare and deliver the tool to the point of use. Effective and efficient tool crib management is therefore critical to the quality and speed of tool change. In this respect, simulation does not only allow the sizing of tool cribs, but also the management of tool crib staff and equipment requirements to support the process.

Tools are perishable assets yet costly. Monitoring the tool usage is therefore critical to ensure that the tools are only replaced just before the end of their life. If replaced too late, then the quality of the part pieces being machined (e.g., cylinder blocks or heads) would obviously be affected; and the CNC machines would consume more energy, as worn tools would increase the cycle time of the machine and ultimately in the long run, could damage the machine. Changing tools too early, on the other hand, simply means waste, which is contradictory to the concerted effort by the management on reducing the tool inventory due to the high and increasing cost of tools.

Tool crib's operators typically work 3 shifts with 2 operators per shift. As the operators are not unlimited resources, they are critical to the performance of the tool delivery. Timely tool delivery scheduling ensures the availability of the tool crib's operators to dispatch the required tools to the lines. On the line, the CNC operators are also required to keep track of the tool change schedule. In most cases, they are also required to check or re-set the tools, in addition to carry out the tool change and other maintenance regimes.

This paper describes an industrial project that aims to enhance the existing simulation modeling suites at a car engine factory in the UK with a new feature that can effectively model the tool delivery process focusing on how the tool crib operates, how tools are supplied to the lines and various operating strategies. The project involved an in-depth investigation into the tool delivery to the machining lines and the tool crib feeding them. The deliverables of the project seeks to enhance the company's simulation suites that can be extensively used by the tooling engineers, allowing them to evaluate and experiment the different scenarios and strategies of tooling management before actual implementation.

\section{TOOL CRIB MODEL}

Initial design of the tool crib was made possible by carrying out an in-depth observation in the tool crib and by interviewing tooling engineers and operators in order to understand the actual processes to be modeled. Modifications and updates were made to the model due to amendments to the process flow analysis, to ensure that it sufficiently represents the current state of the tool crib and how it delivers tools to the machining lines. The model was built based on the process flow and in agreement with the tooling engineers.

\subsection{Basic Model}

The simulation model of tool crib was built in Witness ${ }^{\circledR}$ with the details gathered at the process analysis stage. Equipment inside the tool crib that was modeled include two visual gauges, two heat shrinks, one contact gauge, one wash machine and workbenches. Basic modeling components used in the model are shown in Table 1.

For each machine, a set of operations were performed according to the properties of the tools when they arrive. The tools are represented by entities (or parts in Witness ${ }^{\circledR}$ term), split into 15 different categories. This has reduced complexity of the model in terms of tools routing in the tool crib. These categories were continually reviewed during the project to accommodate the vast number of tools.

An attribute was assigned to each part every time it is created in order to identify its category. The initial value was given randomly using the Integer Uniform distribution. Only 8 of the first 15 categories belong to the cylinder block and cylinder head production lines. Afterwards, the different routes for the different categories were created. To verify the logic of the part route, only one category of part was as- 


\section{Tjahjono and Ladbrook}

signed at a time. The process route inside the tool crib was stepped through. A variable was created to store the values of the tool category attribute and to plot a pie chart for the tool usage.

Table 1: Witness ${ }^{\circledR}$ components used to model the tool crib

\begin{tabular}{ll}
\hline Element & Description \\
\hline Entity/Part & Represents the tool to be processed \\
Machine & Represents an equipment inside the tool crib (visual gauge, crank gauge, etc) \\
Buffer & Stores tools \\
Vehicle & Transports tools from the tool crib to lines \\
Track & Guides the vehicle \\
Variable & Stores information in the model \\
Attribute & Stores information in a part \\
Module & Group of elements working as a sub-model inside the main simulation model \\
\hline
\end{tabular}

The time each tool spends in each machine was calculated as follows:

- Wash machine is a fixed time of less than a minute.

- Heat shrink is also a fixed time, but has two operations. The machines can be of two types, working with hot water (needs cool down) or automatic (does not need cool down, much quicker). The possibility of using each of them is implemented in the model.

- Cycle times for Visual Gauge, Contact Gauge, Workbench and Crank Gauge were calculated considering the total available times, the fixed operations and the number of operations without cycle times.

- Some tools have to go first to the Visual Gauge to get their tag read (fixed cycle time).

The iterative approach adopted in building the model significantly helped in identifying errors and bugs hence allowing effective model development.

\subsection{Integration with the Existing Simulation Suite}

The existing simulation suite used in this project is essentially a proprietary Excel spreadsheet that acts as an input/output interface to Witness ${ }^{\circledR}$. The spreadsheet has many features, one of which being the automatic model generation. The user interface significantly speeds up model building, making it easier for manufacturing engineers who are not necessarily familiar with simulation modeling, to build and modify complex machining line models (Benedettini and Tjahjono 2009). Macros inside Excel allow the automatic code generation by linking together the library of custom designed Witness ${ }^{\circledR}$ modules. The library of modules consists of machines and conveyors typically present in a machining line.

At present, the factory simulation suite models tool changes by including the durations of the tool changes inside the model as variables. Several variables were used to store the information of different change frequencies of the tools; every time a part was produced by the CNC machine, all the tools involved in its production reduce their life counters by one unit. Once the countdown of a tool reached zero, a setup time was included as an addition to the cycle time of the machine and the life of the tool being reset. It was assumed that spare tools were always available inside the model.

The tool crib model that has been integrated into the existing factory simulation suite enables more accurate representation of the tool change (using parts as opposed to variables) resulting in more accurate prediction of the tool life. The carrousel of the CNC cell is represented using a buffer where the exchange between the new and old tools takes place, and the CNC cells inside the Witness ${ }^{\circledR}$ module are represented using two machines. One of them is designed to represent the physical distribution of parts between the different machines acting as the gantry of a $\mathrm{CNC}$ cell; and the other is an element where the parts are sent 


\section{Tjahjono and Ladbrook}

to (and represents the manufacturing of a part), which takes into account the procedure to adjust the tool countdown.

Figure 1 shows the screenshot of the simulation suite and the tool crib model developed.

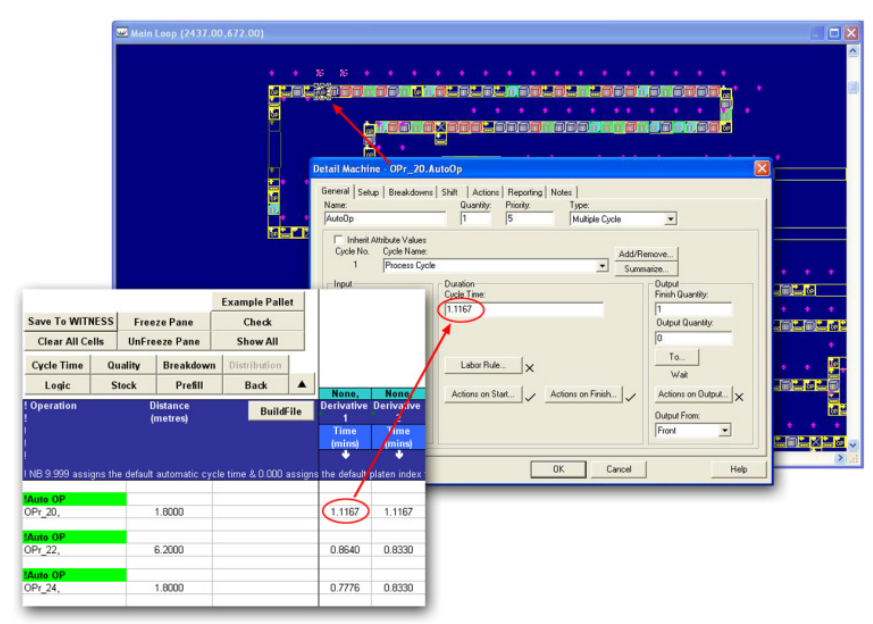

(a)

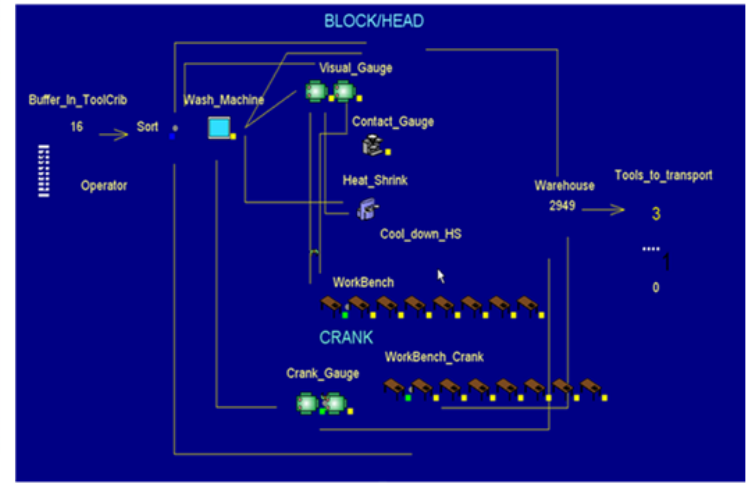

(b)

Figure 1: Screenshots of (a) the simulation suite; (b) the tool crib model

The remaining number of parts (i.e., cylinder heads or blocks) to be produced with a tool, set a warning level. Once the life of one tool goes under this level, the system automatically asks for a new tool and the next two tools, whose life times are closest to the warning limit, to be replaced. Every time the new tools are delivered to the system, they are installed at the carrousel for the production of one part to be sent to a manual checking station. Once the production of this part is completed, the machine is set to use the old tools again while the new ones wait at the carrousel. The final change of the used tool for a new one only takes place when it reaches the end of its life and the part produced by the spare tool has already passed quality check.

The tool change process inside the model is divided into three sub-stages: 1) demand of tools, 2) production of a part with new tools, and 3) change of tools. Whenever a part enters the CNC cell in the mod$\mathrm{el}$, it calculates if there is any tool that has been through the warning level. In the situation that the system detects at least one tool under the warning level, it enters a loop of prioritization to obtain the next two tools with the shortest remaining life.

The next step is designed to find out if the tools are at the warehouse. Once the tool with higher priority is available, a flag is triggered to activate their extraction from the tool crib warehouse. This is also used to transport the tool with the second and third priority following a sequential logic; as such the third tool should not be asked for if the second is not available. The tools extracted from the warehouse are stored in a buffer where they wait for dispatch.

The tool crib and the CNC cells are two different modules inside the Witness ${ }^{\circledR}$ simulation. The exchange of information from the tool crib to the CNC cells (and vice versa) is done through variables located in a simulation folder in the system. The trigger used to activate the process also ensures that the value of the variables to store the information of the tools demanded could not be rewritten by other elements in the system.

Since the conditions to order tools will be fulfilled during several cycles in order to avoid the continuous demand of tools, a simple locking feature was implemented to allow only one demand of tools at a time. Only when the set of tools demanded by the machine is delivered to the CNC machine, the system will allow the machine to make another demand. Once delivered, it activates a trigger to move these tools to the buffer where the spare tools are stored, and at the same time, sets the destination of the part-piece (i.e., cylinder head or block) produced to manual checking. The traceability is assured by the continuous 


\section{Tjahjono and Ladbrook}

update of different variables in the model to store information on which spare tools has the machine produced and checked.

The final step of the tool management algorithm is the change of a tool with no more remaining partpieces to produce. This involves two actions: the transportation of the used tools to the side cabinet for collection and the allocation of a new tool at the carrousel. Variables and elements performing these operations are included in the CNC module. Due to the availability of different machines per module, the locking strategy to avoid the rewriting of the trigger and variables involved in the transportation was taken into consideration and logic was rewritten to tackle scenarios such as, when the probability of events were low and several tools can be changed simultaneously.

One of the important modifications made to the simulation suite was the mechanism to stop the CNC machines when the spare tools are not present and their reactivation when the tools arrive. Two different circumstances could lead to a blockage of the CNC cell, the absence of a spare tool and a delay on its quality checking confirmation. The blockage of a line due to the lack of tools was not considered in the existing models. The algorithm managing the tool change was completely dependent on the continuous manufacturing of the CNC cells. In order to enhance the existing algorithm, an external capability was added to perform all the tool delivery operations even in the case that the machining line is stopped. Emulating the process at the factory, this system will repeatedly demand the tools, and once delivered, it activates the machine again. The main advantage of this is the possibility to measure the lost throughput during stoppage of CNC machines which is in essence a bottleneck of the machining line.

The exchange of tools between the different CNC cells and the tool crib has been modeled using tracks and vehicles in Witness ${ }^{\circledR}$. At the CNC module, two tracks have been included to simulate the exchange of tools at the side cabinets. The vehicle (or also known as milk run), which is located at the tool crib, periodically covers a route of different $\mathrm{CNC}$ cells delivering the tools demanded and collecting the used ones. As the demand of tools varies, the regularity of the milk run is ensured with a time counter. If the duration of the delivery is high, then the idle time of the vehicle is reduced proportionally.

In order to deal with the fact that Witness ${ }^{\circledR}$ does not allow the use of an attribute as an unloading condition for all the parts inside a vehicle and the distribution of the parts demanded by a specific CNC, a variable was used at the beginning of each track to calculate how many tools should be delivered. The tools on a vehicle are disposed as a pile. The track reads the values of the attributes containing the destination, and starts to unload the quantity calculated from the first tool that satisfies the condition. The inclusion of these elements has been an a significant contribution to enhance the existing simulation suite.

\section{EXPERIMENTATION}

The tool crib model was built to represent the actual tool crib in the engine plant in terms of layout but the configuration of the equipment inside the crib and number of tooling operators may not be the optimal combination that could meet the tooling requirements of the line. The aims of the experiments are to obtain the most optimal configuration of resources available in the tool crib that give the most timely delivery of tools to the lines and at the same time to reduce the tool inventory level.

\subsection{Experiment 1: Configuration of the Tool Crib Resources}

For the purpose of designing reasonable scenarios, the experimentation was split into two stages. The first stage is to find the scope for the reasonable scenarios, which will not include many idle machines and the second stage is to carry out the experimentation within that scope.

The tool crib outputs are obviously influenced by resources (machines and people) in the tool crib. However, the range can vary for different resources, therefore a sensitivity analysis after each scenario will be done to find the best scenario. For instance, if the tools waiting at the entrance of the tool crib keep increasing, then that experiment will be stopped and the model will be reset to move on to the next reasonable scenario. On the contrary, if the tools inventory decreases to near zero before next delivery, then that experiment can be used for further analysis. 


\section{Tjahjono and Ladbrook}

The data used for the experimentation are:

- number of tools sent to the tool crib

- number of tools waiting at the entrance of the tool crib

- number of operators; machine's busy, idle, blocked and waiting times

- number of tools sent to the warehouse

- number of tools stored in the warehouse

Table 2 shows the best scenario analysis listing the comparison of the 6 scenarios with different configuration of input data parameters.

The results indicated that the best scenario is given by a combination of 4 operators, 1 contact gauge, 1 heat shrink, 2 visual gauges and 2 crank gauges. There is no significant difference between the total number of tools that were sent to the tool crib and the tools waiting at the entrance of the tool crib under this combination.

\subsection{Experiment 2: Tool Inventory Level}

This experiment investigates the impact of the tool crib configuration to the tool inventory level. Inventory level obviously indicates the level of investment that whenever possible should be minimized aligned with the concerted effort by the management on reducing the tool inventory due to the high and increasing cost of tools.

The question this experiment will answer is therefore: "what is the configuration of the resources in the tool crib that can minimize the tool inventory but at the same time give maximum throughput of the line?" The aim of this experiment is therefore to determine the minimal inventory level of tools to hold inside the tool crib warehouse.

In this experiment, the inventory level in previous experiment is required. Once the right level of inventory for every tool warehouse is chosen, it is important to look out for their fluctuations. To obtain the suitable stock for the comparison between scenarios, the following steps were followed:

- Run scenarios with very high level of tool inventory that already exists in each warehouse.

- Statistically study the fluctuations (as normally distributed).

- Check the minimum level of inventory during the simulation time.

- Compare this value with the average of the inventory level (minus 3 times standard deviation).

- Remove from the initial inventory level, the minimum of these two values.

- Run the experiment again and check that the performance of the tool crib does not change.

The above procedure was carried out for each tool (135 analyzed in total) achieving minimum inventory level for every scenario. The data for the comparison were the sum of each tool warehouse (considering the total number of tools inside the tool crib warehouse). This method is arguably not the one that minimize the inventory for every scenario, but the method is believed to be suitable for the purpose of the study. Table 3 shows the configuration of the resources in the tool crib in terms of number of operators, visual gauge and crank gauge.

\section{DISCUSSION AND CONCLUSION}

Using the tool crib simulation model to determine the optimum configuration of equipment, labor and the tool inventory level in the tool crib, it is possible for the management to identify what affects the performance of the machining lines fed by the tool crib, hence the overall production. The experiments were carried out to identify the best economical and feasible scenarios to configure future tool crib to serve the lines. The implications from the model building and experimentation conducted are discussed below. 


\section{Tjahjono and Ladbrook}

Table 2: Best scenario analysis

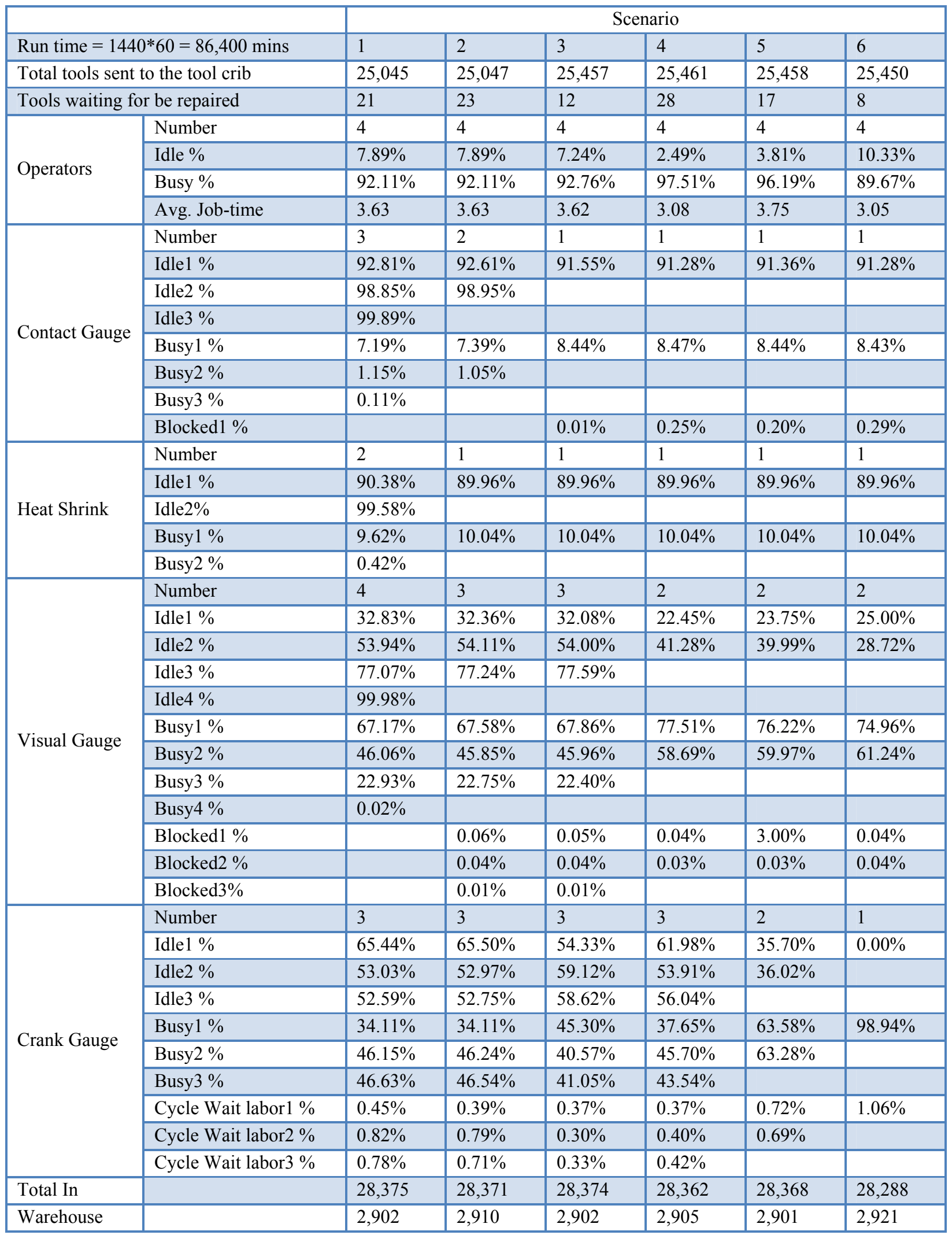




\section{Tjahjono and Ladbrook}

Table 3: Input parameters to obtain the optimum tool inventory level

\begin{tabular}{cccc}
\hline Scenarios & Operators & Visual Gauge & Crank Gauge \\
\hline 1 (Initial) & 4 & 2 & 2 \\
\hline 2 & 4 & 2 & 3 \\
3 & 4 & 3 & 2 \\
4 & 4 & 3 & 3 \\
5 & 5 & 2 & 2 \\
6 & 5 & 2 & 3 \\
7 & 5 & 3 & 2 \\
8 & 5 & 3 & 3 \\
9 & 6 & 2 & 2 \\
10 & 6 & 2 & 3 \\
11 & 6 & 3 & 2 \\
\hline 12 & 6 & 3 & 3 \\
\hline
\end{tabular}

The results of each scenario are plotted in Figure 2 below:

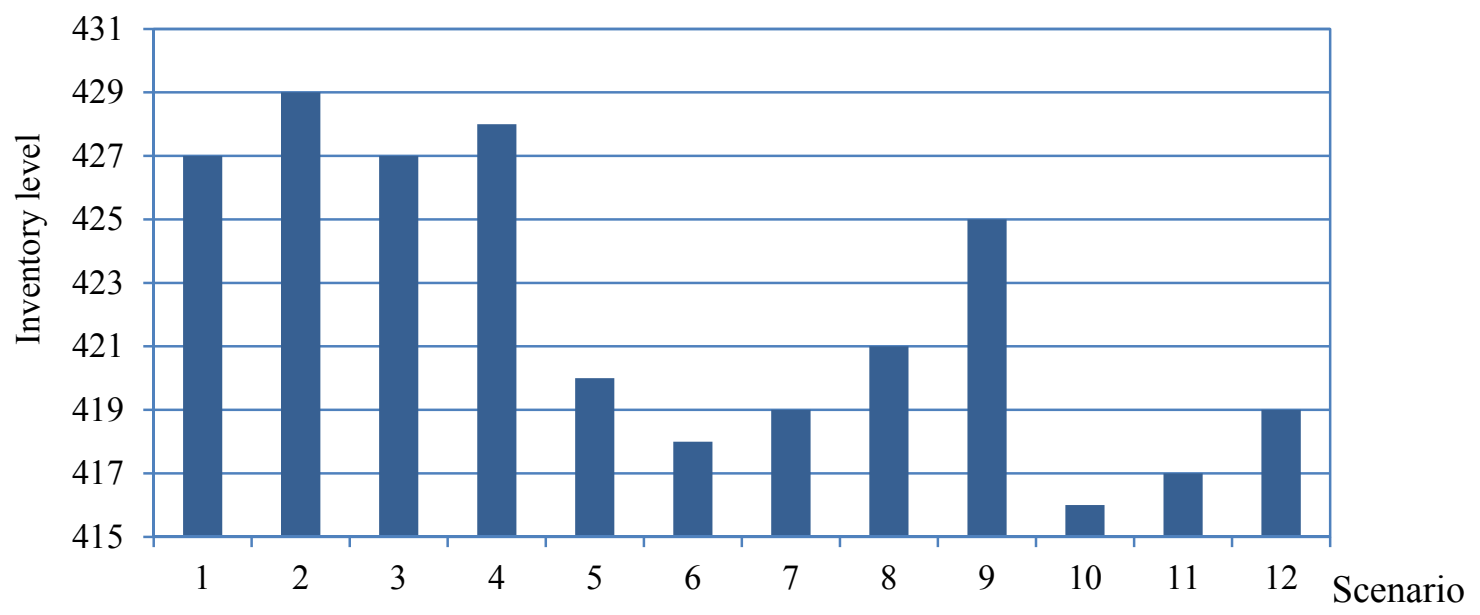

Figure 2: Inventory levels for each configuration

\subsection{Configuration Mixes}

By categorizing the tools that most frequently arrive into the tool crib and studying their relationship with the equipment in the crib (e.g., visual gauge, crank gauge, etc.), it is possible to identify the best combination of these resources to meet each planned capacity for a tool crib. From the experiments, it was found that the crank gauge was the key constraint of the tool crib. Possible explanation is that although not many tools are processed by this equipment, the time taken to gauge the tool is much longer than that in any other equipment in the crib, during which the tool crib operators were fully occupied at this equipment, leaving other tools arriving at the same time waiting.

The experiments also revealed the various scenarios for an ideal tool crib with minimal resources that still meet the tool delivery requirements. This is the best scenario in terms of efficiency where the numbers of tools are serviced in the tool crib and then sent to the warehouse are considerably higher than that in the other scenarios. All four operators are also fairly distributed to service the used tools arriving to the tool crib. 


\section{Tjahjono and Ladbrook}

\subsection{Operators}

It is vitally important to man the tool crib effectively and consequently to determine the most economical level of staffing. At present, typically two operators are sufficient to man the tool crib but some of the crank tools could take up to three hours (excluding waiting time) to gauge. Therefore, it is critical to firstly consider the size of the tool crib and then the number of operators necessary to cover the crank and all other tools. The experiments conducted showed that to effectively deal with the constraints of the crank gauge, it is suggested to have a minimum of four operators, ideally two operators dedicated to rework the crank tools and the other two on other tool types. If the servicing can be done outside the tool crib, then the two operators could run the tool delivery system.

\subsection{Inventory Level}

The experiment also suggested that it is possible to reduce the tools inventory level with minimal adverse effect on the machining lines. Six operators, three visual gauges and two crank gauges could be chosen for this purpose. The inventory level cost is a function of ordering and holding costs, and considering that every other scenario includes more machines or more workers, that means that in terms of stock level, increasing the size of the tool crib does not seem to make any difference. The initial tool crib warehouse has passed this test giving the best alternative design.

\subsection{Improvement to the Existing Simulation Suite}

The modification to the existing simulation modeling suite offers the scalability to automatically create the tool cribs and the different stations that construct a machining line and its complete configuration. The low-level command inside the simulation suite's user interface has been significantly modified to include the option to build up to nine different tool cribs allowing all their parameters and configuration to be defined by the users. Individual customized tool cribs could be built to represent all the different existing facilities at the plant, by changing the number of operators, storage capacities, tool delivery times, the selection of processes and their duration, etc. In addition to that, the tracks and vehicles at the CNC module enables the development of different scenarios to determine the different routes for each tool crib. A function was programmed to read the input destinations and then automatically create the logic in the simulation suite to link the tool crib vehicles and the tracks of the $\mathrm{CNC}$ modules.

\subsection{Concluding Remarks}

The work described in this paper is part of an on-going project towards achieving the 'total plant modeling' at the sponsoring company. The main deliverable of this project was a tool delivery system in the form of an integrated machining line and tool crib model. The model allows tooling engineers to decide the most effective tooling strategy to plan the future tool crib configuration. Based on the experiments carried out, the best configuration of the tool crib has been identified in order to achieve the best performance of the entire machining line in terms of production throughput whilst maintaining minimal tool inventory level. To maintain timely delivery of tools to the lines, it is critical to effectively prioritize the planning of the tool crib. Better management of tooling operators is also necessary in order to reduce the bottleneck inside the tool crib. One suggestion that can be made is that some complicated and time consuming operations could be outsourced and only a certain types of tool will be stocked on site. This suggestion requires further investigation.

\section{ACKNOWLEDGMENTS}

The following individuals are part of the project team and their contributions to the work reported herein are gratefully acknowledged: Carmen Carbonell, Heng Jing, Alfred Ampomah Kusi, Roberto Pasqualino, Zhang Wei and Luis Zurro. 


\title{
REFERENCES
}

Baines, T. S., and J. M. Kay. 2002. "Human Performance Modelling as an Aid in Process of Manufacturing System Design: A Pilot Study." International Journal of Production Research 40: 2321-2334.

Benedettini, O., and B. Tjahjono. 2009. "Towards an Improved Tool to Facilitate Simulation Modelling of Complex Manufacturing Systems." International Journal of Advanced Manufacturing Technology 43: 191-199.

Ohno, T. 1988. Toyota Production System: Beyond Large-Scale Production. Productivity Press.

\begin{abstract}
AUTHOR BIOGRAPHIES
BENNY TJAHJONO is a Senior Lecturer at the Manufacturing Department, School of Applied Sciences, Cranfield University. He received MSc and $\mathrm{PhD}$ degrees from Cranfield. His research interests are in the application of modeling and simulation of manufacturing systems. He has been working closely with global companies such as Rolls-Royce, Airbus and Motorola on a number of industrial projects. He is an associate editor of the Journal of Simulation and a member of the editorial board of several journals. He can be reached at b.tjahjono@cranfield.ac.uk.
\end{abstract}

JOHN LADBROOK is a Visiting Fellow at the Manufacturing Department, Cranfield University. His MPhil degree from University of Birmingham concerns with modeling breakdown within manufacturing systems. His hands-on experience in manufacturing simulation dated back to early eighties when he first introduced the use of discrete-event simulation as part of decision making process in the company he worked for. His interest is in promoting the use of simulation by non-simulation expert, hence increasing the uptake of the technique within manufacturing companies. He can be contacted at j.ladbrook@cranfield.ac.uk. 